\title{
Características radiológicas de la hematopoyesis extramedular. A propósito de un caso
}

\section{Radiological Characteristics of Extramedular Hematopoyesis. About a Case}

\author{
Pilar Márquez Sánchez ${ }^{1}$ Francisco M. Páez Codeso ${ }^{2}$ María Carmen Fernández Marín²
}

1 Servicio de Radiodiagnóstico, Hospital Regional de Málaga, España

${ }^{2}$ Servicio de Neumología, Hospital Regional de Málaga, España

Rev Argent Radiol 2019;83:170-172.

Estimados Editores:

La hematopoyesis extramedular (HEM) en el adulto consiste en el desarrollo de tejido hematopoyético ectópico para compensar una eritropoyesis ineficaz. ${ }^{1,2}$ Es una enfermedad inhabitual y ha sido más frecuentemente reportada en pacientes con anemia crónica severa y talasemia, pero también en pacientes con leucemia, hemoglobinopatías y mielofibrosis. ${ }^{3}$ Los lugares más comunes de HEM son el hígado y el bazo, aunque pueden encontrarse focos en numerosos tejidos, incluyendo las áreas paravertebrales del tórax. Presentamos el caso de una paciente de 67 años que consultó por disnea, objetivándose en la radiografía (RX) de tórax inicial unas masas paravertebrales, cuyo diagnóstico final fue HEM. Nuestro objetivo es la evaluación de las características imagenológicas más frecuentes de la HEM.

Se trata de una paciente con antecedentes personales de anemia crónica por microesferocitosis hereditaria diagnosticada en 1998, tratada con hierro y ácido fólico.

En julio de 2015 consultó en el servicio de Neumonología por disnea moderada al esfuerzo y tos seca. El laboratorio objetivó anemia leve con hemoglobina de 10,3 g/dl, reticulocitosis elevada con índice de dispersión de hematíes de $17,9 \%$, ferritina de $539 \mathrm{ng} / \mathrm{ml}$ y bilirrubina total de $1,81 \mathrm{mg} /$ dl. El estudio funcional con espirometría, pletismografía y test de la marcha de 6 minutos fue normal. En la RX de tórax inicial se apreció un desplazamiento de las líneas paravertebrales con una imagen nodular en el hemitórax izquierdo ( - Fig. 1), por lo que se completó con una tomografía computarizada (TC) de tórax (-Fig. 2), donde se identificaron varias masas sólidas paravertebrales, bien delimitadas en el mediastino posterior, entre D9 y D11, isodensas con respecto al músculo y con pequeños focos de grasa.

received

February 8, 2018

accepted

May 8, 2019
Address for correspondence Francisco M. Páez Codeso, Servicio de Neumología, Hospital Regional de Málaga, calle Manzanilla 13, CP 29190, Málaga, España (e-mail: paezco64@gmail.com).

Se completó con una resonancia magnética (RM) de la columna dorsal con administración de gadolinio intravenoso (-Fig. 3), que mostró varias masas bien delimitadas, lobuladas, bilaterales, en mediastino posterior entre D9 y D11, con señal heterogénea hipo e hiperintensa en secuencias ponderadas en $\mathrm{T} 1$, iso e hiperintensa en $\mathrm{T} 2$ y STIR, variables a causa de la presencia de zonas con médula ósea (MO) hematopoyética y zonas con MO grasa, y con captación de contraste I.V. moderado e inhomogéneo. Las características radiológicas de esas masas, con el antecedente hematológico, orientaron el diagnóstico hacia focos de hematopoyesis extramedular. Dos años después, la

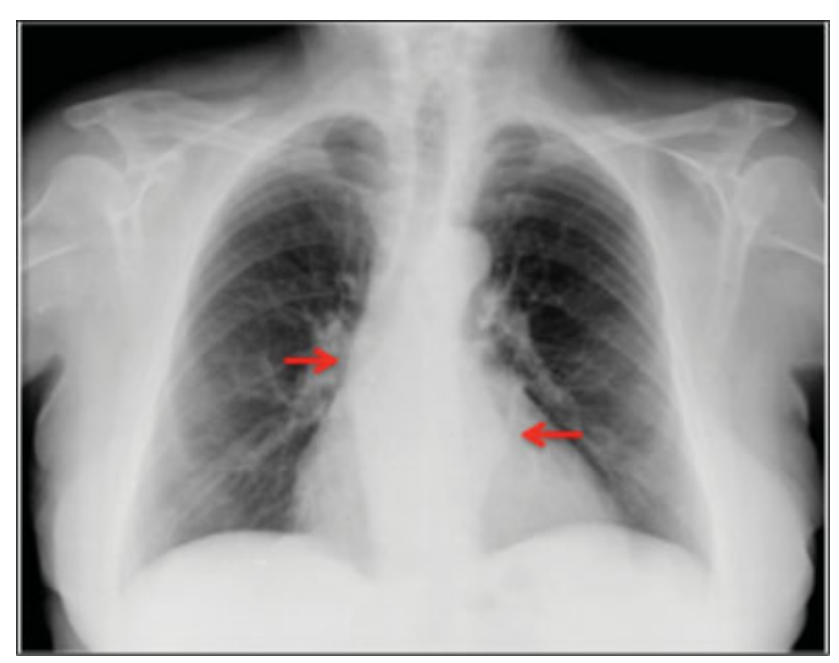

Fig. 1 RX ántero-posterior de tórax. Desplazamiento de las líneas paravertebrales (flechas rojas) más prominente en la izquierda.
Copyright @ 2019, Sociedad Argentina de Radiología. Publicado por Thieme Revinter Publicações Ltda., Rio de Janeiro, Brazil. Todos los derechos reservados.

\section{License terms}

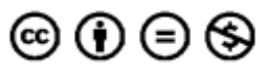




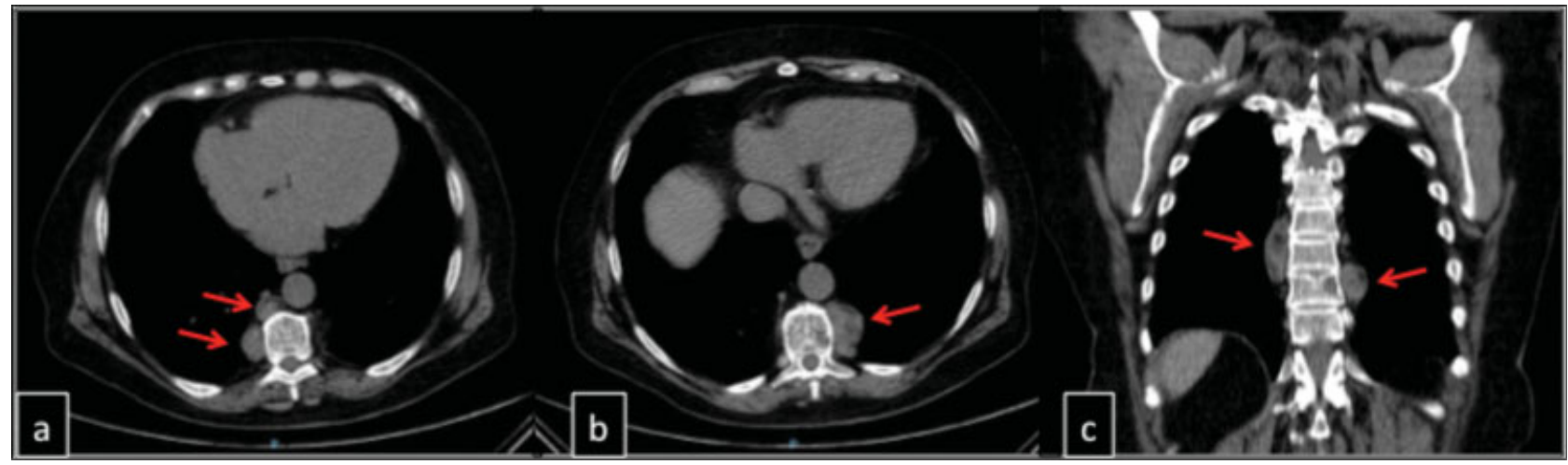

Fig. 2 TC sin contraste I.V. Cortes axiales (a y b) y reconstrucción coronal (c). Se observan varias masas en el espacio mediastínico posterior (flechas rojas) con densidad de tejidos blandos y contenido graso.
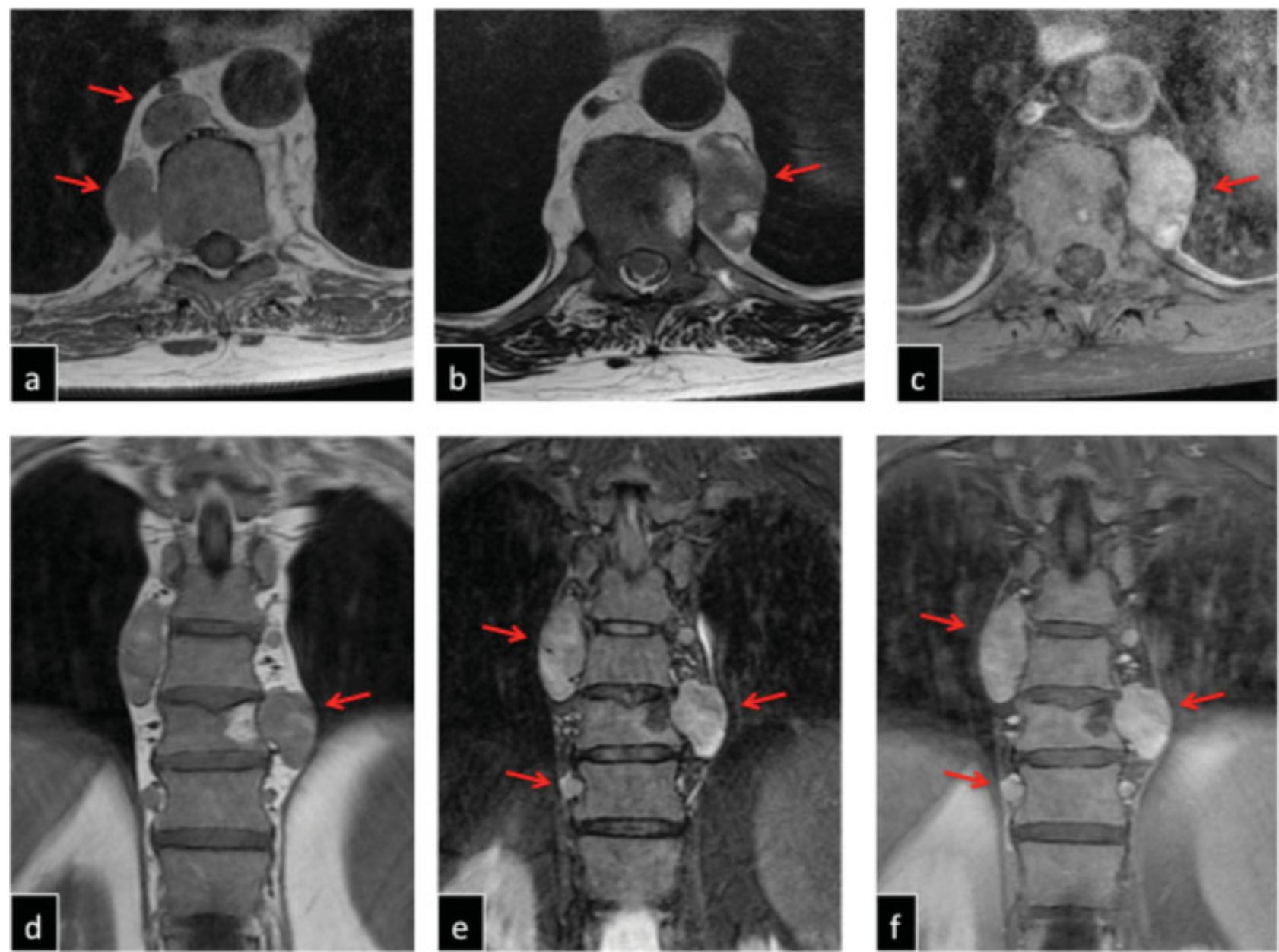

Fig. 3 RM dorsal con Gd. Secuencias en plano axial ponderadas en T1 (a), T2 (b), T1 con Gd y saturación de la grasa (c), coronal ponderado en T1 (d), STIR (e) y T1 con Gd y saturación grasa (f). Se observan varias masas paravertebrales (flechas rojas) entre D9 y D11 compatibles con focos de HEM, de señal heterogénea, hipo, iso e hiperintensa ponderada en T1, moderadamente isointensa con focos hiperintensos ponderados en T2, hiperintensa en STIR y con captación homogénea de Gd.

tomografía computada (TC) de control no mostraba cambios en las lesiones.

La HEM consiste en la formación de células sanguíneas fuera de la MO. Durante el desarrollo embrionario hay una HEM fisiológica en el hígado y bazo. Sin embargo, si durante la vida adulta se produce una hematopoyesis ineficaz o una hemólisis incrementada ocasionada por enfermedades hematológicas (anemia crónica, drepanocitosis, talasemia, hemoglobinopatías, leucemia, mielofibrosis, etc.), se desarrolla un mecanismo compensador, que en un principio transforma la MO amarilla (grasa) en médula hematopoyética. Si ese mecanismo también es insuficiente para mantener una hematopoyesis eficaz, se fuerza la expansión de tejido hemopoyético fuera de la MO. Los 
focos localizados de HEM más frecuentes en el adulto se originan en células pluripotenciales hepáticas y esplénicas (95\% de casos), pero también es posible su desarrollo ocasional en ganglios linfáticos, pleura, pulmones, pared bronquial, tracto gastrointestinal, piel, duramadre, riñones, glándulas suprarrenales, etc. ${ }^{4,5}$ Se pueden producir masas en mediastino posterior, paravertebrales, sobre todo en la región dorsal, siendo esa la localización más frecuente de la HEM no hepato-esplénica. ${ }^{2,5}$

En la RX simple de tórax se identifica un desplazamiento de las líneas paravertebrales, con frecuencia bilateral. ${ }^{6}$ En la TC, esas masas muestran una señal isodensa con respecto al músculo, algo heterogénea, con contenido graso en proporción variable, contornos lisos y bien delimitados, sin erosión ósea y con moderada captación de contraste. ${ }^{7}$ La RM muestra habitualmente masas paravertebrales, sobre todo en el segmento dorsal, frecuentemente bilaterales, con intensidad de señal variable debido al componente graso. ${ }^{8}$ Esas masas suelen tener una señal hipo o intermedia y heterogénea ponderada en T1, moderadamente hipointensa con focos hiperintensos en T2 y STIR, y captación de Gd moderada o ausente. ${ }^{9}$ Sin embargo, si ellas muestran un componente graso, la intensidad de la señal será hiperintensa en secuencias potenciadas en T1 y T2. $^{7}$

El diagnóstico definitivo se realiza con toma de biopsia guiada por TC, aunque ese procedimiento tiene un alto riesgo de hemorragia, por lo que la realización de TC y/o RM, que permiten definir los focos de HEM y su contenido graso, se han convertido actualmente en el gold standard de diagnóstico como técnicas no invasivas, junto con características clínicas que hagan sospechar la patología. ${ }^{10}$

Confidencialidad de los datos

Los autores declaran que han seguido los protocolos de su centro de trabajo sobre la publicación de datos de pacientes y que todos los pacientes incluidos en el estudio han recibido información suficiente y han dado su consentimiento informado por escrito para participar en dicho estudio.

Conflicto de Intereses

Los autores del trabajo declaran no tener ningún conflicto de intereses.

\section{Bibliografia}

1 Rosada J, Bindi M, Pinelli M, Pandolfo C, Cassetti G, Castiglioni M. Extramedullary hematopoyesis: compensatory mechanism or clinic syndrome? Case report and review of literature. Med Interna 2007;24(02):77-80

2 Delavaud C, Lincot J, Debray MP, Schouman-Claeys E, Dallaudière B. Paravertebral extramedullary hematopoiesis. Diagn Interv Imaging 2014;95(04):457-460

3 Ginzel AW, Kransdorf MJ, Peterson JJ, Garner HW, Murphey MD. Mass-like extramedullary hematopoiesis: imaging features. Skeletal Radiol 2012;41:911-916

4 Sawada H, Higuchi T, Koyamada R, Okada S. Myelodysplastic syndrome developing presacral extramedullary hematopiesis with atypical MRI findings. Intern Med 2017;56(10):1213-1217

5 Isik Balci Y, Kaya V, Atesçi MS. Presacral and intrathoracic extramedullary hematopoiesis: a case report. Clin Imaging 2012;36(04):406-408

6 Zhu G, Wu X, Zhang X, Wu M, Zeng Q, Li X. Clinical and imaging findings in thalassemia patients with extramedullary hematopoiesis. Clin Imaging 2012;36(05):475-482

7 Orphanidou-Vlachou E. Tziakouri- Shiakalli C, Georgiades CS. Extramedullary hemopoiesis. Semin Ultrasound CT MR 2014;35 (03):255-262

8 Roberts AS, Shetty AS, Mellnick VM, Pickhardt PJ, Bhalla S, Menias CO. Extramedullary haematopoiesis: radiological imaging features. Clin Radiol 2016;71(09):807-814

9 Tsitouridis J, Stamos S, Hassapopoulou E, Tsitouridis K, Nikolopoulos P. Extramedular paraspinalhematopoiesis in thalassemia: CT and MRI evaluation. Eur J Radiol 1999;30(01):33-38

10 Bukhari SS, Junaid M, Rashid MU. Thalassemia extramedullary hematopoiesis, and spinal cord compression: A case report. Surg Neurol Int 2016;7(05):S148-S152 